\title{
Exact Calculation of Blocking Probabilities \\ for Bufferless Optical Burst Switched Links with Partial Wavelength Conversion*
}

\author{
Nail Akar and Ezhan Karasan \\ Department of Electrical and Electronics Engineering \\ Bilkent University \\ 06800 Ankara, Turkey
}

\begin{abstract}
In this paper, we study the blocking probabilities in a wavelength division multiplexing-based asynchronous bufferless optical burst switch equipped with a bank of tuneable wavelength converters that is shared per output link. The size of this bank is generally chosen to be less than the number of wavelengths on the link because of the relatively high cost of wavelength converters using current technologies; this case is referred to as partial wavelength conversion in the literature. We present a probabilistic framework for exactly calculating the blocking probabilities. Burst durations are assumed to be exponentially distributed. Burst arrivals are first assumed to be Poisson and later generalized to the more general phase-type distribution. Unlike existing literature based on approximations and/or simulations, we formulate the problem as one of finding the steadystate solution of a continuous-time Markov chain with a block tridiagonal infinitesimal generator. We propose a numerically efficient and stable solution technique based on block tridiagonal LU factorizations. We show that blocking probabilities can exactly and efficiently be found even for very large systems and rare blocking probabilities. Based on the results of this solution technique, we also show how this analysis can be used for provisioning wavelength channels and converters.
\end{abstract}

\section{Introduction}

Optical Burst Switching (OBS) has recently been proposed as a candidate architecture for the next generation optical Internet [17]. In OBS, the reservation request for

${ }^{*}$ This work is supported in part by The Science and Research Council of Turkey (Tübitak) under project no. EEEAG-101E048 and by the Commission of the European Community IST-FP6 e-Photon/ONe project. a burst is signalled out of band and processed in the electronic domain whereas the burst itself is transported in the optical domain, which makes OBS suitable for all optical networking. To reduce delays, transmission of a burst follows the reservation request without having to wait for an acknowledgement of a successful path set-up. In this paper, we consider the Just Enough Time (JET)-based reservation OBS protocol [16]. In this architecture, when the control packet arrives at an Optical Cross Connect (OXC), the switch is configured for accommodating the burst arriving after an offset time if capacity is available at the node. Otherwise the burst is said to be blocked. Allocated resources are released as soon as the burst is transmitted by the OXC. The main goal of this paper is to study such burst blocking probabilities.

To cope with the burst blocking in OBS networks, Wavelength Division Multiplexing (WDM) systems are used so that different wavelengths on the same fiber are treated as parallel links between two optical nodes. Given a WDMbased OXC with a fixed number of wavelengths per I/O fiber line, Tunable Wavelength Converters (TWC) can be used to switch bursts from one wavelength to another for contention resolution. In Full Wavelength Conversion (FWC), a burst arriving at a certain wavelength can be switched onto any other wavelength towards its destination. FWC may reduce blocking probabilities significantly when compared with No Wavelength Conversion (NWC) [1],[9], [11]. However implementing all-optical FWC is very difficult and costly due to technological limitations. Partial Wavelength Conversion (PWC) is proposed as a costconscious alternative to FWC. In PWC, there is a limited number of TWCs, and consequently some bursts cannot be switched towards their destination (and therefore blocked) when all converters are busy despite the availability of free channels on wavelengths different from the incoming wavelength. In PWC, TWCs may be collected as a single converter pool for converter sharing across all fiber lines, which 
is referred to as the Share-Per-Node (SPN) architecture [4]. A simpler architecture allows separate TWC banks per output fiber link and the corresponding solution is called the Share-Per-Link (SPL) architecture [4]. Recently, a similar architecture is proposed in [3] where a bank of TWCs is shared for all bursts coming in on the same input fiber link; such an architecture is called the Share-Per-Input-Line (SPIL) architecture. The case of limited-range conversion for which there is a limit on the range of wavelengths to which a given wavelength can be converted to, is left outside the scope of the current paper. The focus of the paper is on OXCs with PWC using the simpler-to-implement SPL architecture and TWCs with no tuning range limit.

We consider the online blocking model described in [18]. In this model, lightpaths are set up and torn down at the burst level. This is analogous to circuit switched telephone networks establishing and tearing down calls except that average call holding times in such networks are typically in the order of minutes that are much larger than average burst transmission times in optical burst switched networks. In synchronous (slotted) networks, burst lengths are fixed and bursts are assumed to arrive at slot boundaries. Although such models are difficult to implement due to the need for expensive synchronization equipment, they are readily amenable to combinatorial analysis [4]. In asynchronous (i.e., unslotted) networks, burst lengths are not deterministic and bursts arrive asynchronously which nullifies the need for synchronization equipment. However, asynchronous networks require probabilistic methods and are generally more difficult to analyze. For the general PWC case, there is no closed form expression for the blocking probability and analytical results are also rare [14]. In [14], a fixedpoint iteration-based approximate method for PWC for the SPN architecture is mentioned but not detailed. The work in [5] is simulation-based and attempts to find the number of wavelength converters required to attain a desired GoS in terms of blocking rates. In [4], simulations and an approximate analysis are provided for the same problem for the asynchronous case. Poisson and non-Poisson dynamic traffic patterns are simulated in [5] and [19] to show the impact of the burst arrival process on throughput. Simulation is generally a very useful tool since it can be applied to a wide range of models but i) it typically suffers from long run-times, ii) studying rare blocking probabilities may not be feasible, iii) it is computationally costly to use simulations in scenarios that might require iterations (e.g., analysis of networks, design and dimensioning problems, etc.).

In this paper, we study an asynchronous OXC based on SPL and PWC. Under the usual Poisson burst arrival and exponential burst length assumptions, we exactly calculate the burst blocking probabilities for a bufferless OBS link. Unlike existing literature based on approximations and/or simulations, we formulate the problem as one of finding the steady-state solution of a Continuous-Time Markov Chain (CTMC) with a block tridiagonal infinitesimal generator. We propose a numerically efficient and stable solution technique based on block tridiagonal LU factorizations. We show that blocking probabilities can exactly and efficiently be found even for very large systems and rare blocking probabilities. We also generalize our results to the more general Phase-type (PH-type) burst arrivals. Based on the results of this solution technique, we also present a design procedure for provisioning wavelength channels and converters.

The remainder of this paper is organized as follows. Section 2 describes the model with Poisson burst arrivals as well as the solution procedure. The extension to PH-type arrivals is studied in Section 3. Section 4 is devoted to numerical examples. We conclude in the final section.

\section{Mathematical Model}

We study an optical burst switch which has $N$ input and output fiber lines and $K$ wavelength channels per fiber. We assume a wavelength converter bank of size $0 \leq W \leq K$ dedicated to each fiber output line. Bursts destined to a particular output fiber line arrive at the switch from any one of the $N$ fiber input lines, the wavelength channel they arrive on is uniformly distributed on $(1, K)$, and burst durations are assumed to be exponentially distributed with mean $1 / \mu$.

A new burst arriving at the switch on wavelength $w$ and destined to output line $k$

- is forwarded to output line $k$ without using a TWC if channel $w$ is available, else

- is forwarded to output line $k$ using one of the free TWCs in the converter bank and using one of the free wavelength channels selected at random, else

- is blocked.

We concentrate on a certain output fiber link $k$ throughout this paper and study its burst blocking performance. The overall switch performance can then be obtained using the individual output fiber blocking probabilities. This is because of the way TWCs are shared (i.e., SPL architecture with complete TWC partitioning across output fiber lines). We first assume that the aggregate burst arrival process from the $N$ input lines and destined for output line $k$ is Poisson with rate $\lambda$. This assumption is very common especially when $N$ is large. For $W=K$ (i.e., full wavelength conversion), the OBS system behaves as an $M / M / c / c$ loss system with $c=K$ servers and offered load $r=\lambda / \mu$. For $W=0$ (i.e, no wavelength conversion), we have $K$ independent single server $M / M / 1 / 1$ loss systems each with offered load $r=\lambda / \mu K$. In an $M / M / c / c$ loss system, burst 
blocking probability (denoted by $P_{b}$ ) can be obtained using the Erlang B formula [7]:

$$
P_{b}=\frac{r^{c} / c !}{\sum_{i=0}^{c} r^{i} / i !}
$$

To the best of our knowledge, PWC or equivalently the case of $0<W<K$ does not have an exact analytical solution in the literature. In order to obtain an exact analytical method, we pursue a Markovian analysis described below. Let $i(t)$ and $j(t)$ denote the number of wavelength channels and the number of TWCs that are in use at time $t$, respectively. The process $X(t)=\{(i(t), j(t))$ : $t \geq 0\}$ is actually a Markov process on the state space $S=\{(i, j): 0 \leq i \leq K, 0 \leq j \leq \min (i, W)\}$. To show this, let us assume that the process is in some state $(i, j), 0 \leq i<K, 0 \leq j \leq \min (i, W)$ at time $t$. If a new burst arrives in the interval $(t, t+\delta)$ which occurs with probability $\lambda \delta+o(\delta)$ (i.e., $\lim _{\delta \rightarrow 0} o(\delta) / \delta=0$ ) [10], there are three possibilities:

A1) the wavelength on which the burst is riding is not currently used on the link which occurs with probability $(K-i) / K$ and the burst will be admitted and the process will jump to $(i+1, j)$ at time $t+\delta$,

A2) that wavelength is already used with occurs with probability $i / K$

- then if $j=W$ then the burst will be blocked because the converter pool is all busy leading to no state change,

- else if $j<W$ then the burst will be admitted on one of the available wavelengths randomly using one of the free converters and the process will make a transition to state $(i+1, j+1)$ at time $t+\delta$.

When the process is in state $(K, j)$ for some $j$ at time $t$, then the arriving burst will be blocked since all wavelengths are busy.

Assume now that the process $X(t)$ is currently in some state $(i, j), 0<i \leq K, 0 \leq j \leq \min (i, W)$ at time $t$. If a burst departs in the interval $(t, t+\delta)$ which occurs with probability $i \mu \delta+o(\delta)$ then there are two possibilities:

B1) a TWC was used for this burst which occurs with probability $j / i$ and the process $X(t)$ will jump to state $(i-1, j-1)$ at at time $t+\delta$,

B2) a TWC was not used at all for this departing burst which occurs with probability $(i-j) / i$ and the process $X(t)$ will make a transition to state $(i-1, j)$ at time $t+\delta$
When the process $X(t)$ is in state $(0,0)$, then there cannot be any departures. It is thus clear that the process $X(t)$ is a CTMC.

The next step is to write the infinitesimal generator of this CTMC in a form that is amenable to numerically stable and efficient computation for the steady-state probabilities. For this purpose, we decompose the state space into subsets called levels such that the number of wavelengths in use is constant within a level and we enumerate the states in the following order:

$$
\begin{aligned}
S=\{ & \underbrace{(0,0)}_{\text {level } 0}, \underbrace{(1,0),(1,1)}_{\text {level } 1}, \underbrace{(2,0),(2,1),(2,2)}_{\text {level } 2}, \cdots, \\
& \underbrace{(K, 0),(K, 1), \cdots,(K, W)}_{\text {level } K}\}
\end{aligned}
$$

Based on this enumeration, we conclude that state transitions can occur either among neighbouring levels or within a level. The final step is to write the infinitesimal generator of the process $X(t)$ based on the observations A1, A2, $\mathrm{B} 1$, and B2. For this purpose, we define the following two matrices for $i \geq 0$

$$
\begin{gathered}
M_{i}=\left[\begin{array}{cccc}
i & & & \\
1 & i-1 & & \\
& 2 & i-2 & \\
& & \ddots & \ddots
\end{array}\right], \\
N_{i}=\left[\begin{array}{cccc}
\frac{K-i}{K} & \frac{i}{K} & \frac{i}{K} & \\
& & \frac{K-i}{K} & \ddots
\end{array}\right] .
\end{gathered}
$$

We let $I_{i}$ and $\bar{I}_{i}$ denote an identity matrix of size $i$ and a zero matrix of size $W+1 \times W+1$ except its last entry which is $i / K$, respectively. The process $X(t)$ is then written as a CTMC with a block-tridiagonal infinitesimal generator $Q$

$$
Q=\left[\begin{array}{ccccc}
A_{0} & U_{1} & & & \\
D_{0} & A_{1} & U_{2} & & \\
& D_{1} & A_{2} & \ddots & \\
& & \ddots & \ddots & U_{K} \\
& & & D_{K-1} & A_{K}
\end{array}\right]
$$

where $A_{i}=-(\lambda+i \mu) I_{i+1}$ for $i<W, A_{i}=-(\lambda+$ $i \mu) I_{W+1}+\lambda \bar{I}_{i}$ for $W \leq i<K$, and $A_{K}=-K \mu I_{W+1}$. Furthermore, $D_{i-1}=\mu \bar{D}_{i-1}$ where $\bar{D}_{i-1}$ is the upper-left $W+1 \times W+1(i+1 \times i)$ block of $M_{i}$ if $i>W$ (if $1 \leq i \leq W)$. Finally, the matrix $U_{i+1}=\lambda \bar{U}_{i+1}$ where $\bar{U}_{i+1}$ is the upper-left $W+1 \times W+1(i+1 \times i+2)$ block of $N_{i}$ if $i \geq W$ (if $0 \leq i<W$ ). Steady-state probabilities 
of this irreducible and aperiodic CTMC can be found by solving for the unique stationary solution (see [10])

$$
\begin{aligned}
x Q & =0, \\
x e & =1,
\end{aligned}
$$

where $e$ is a column vector of ones of suitable size. Note that the size of $Q$ is $(W+1)\left(K-\frac{W}{2}+1\right)$ and calculating the stationary solution using conventional finite Markov chain solution methods (see for example [20]) would have computational complexity $O\left(K^{3} W^{3}\right)$ which might be prohibitive especially for large systems (for example $K=250$, $W>>0$ ). Next we give a numerical solution procedure by taking advantage of the block-tridiagonal structure of the generator.

Since one of the equations in (4) is redundant, we can replace one of the equations, say the first equation in (4), by all ones to obtain a nonsingular system. For this purpose, let $P$ be obtained by replacing the entries of the first column of $Q$ with unity by setting

$$
A_{0}:=1, D_{0}:=\left[\begin{array}{l}
1 \\
1
\end{array}\right]
$$

Also let $b$ be a zero column vector except its first unity entry. It is clear that if $z$ is a solution to

$$
z P=b
$$

then $x:=z /(z e)$ gives the steady-state probabilities.

We propose the block tridiagonal LU factorization algorithm given in [6] for solving $z P=b$. In this algorithm, the goal is to obtain a block LU factorization of the matrix $P$ :

$$
\begin{gathered}
P=\left[\begin{array}{cccccc}
I & & & & \\
L_{0} & I & & & \\
& L_{1} & I & & \\
& & \ddots & \ddots & \\
& & & L_{K-1} & I
\end{array}\right] \times \\
\\
{\left[\begin{array}{cccccc}
F_{0} & U_{1} & & & \\
& F_{1} & U_{2} & & \\
& & F_{2} & \ddots & \\
& & & \ddots & U_{K} \\
& & & & F_{K}
\end{array}\right]}
\end{gathered}
$$

We then partition the solution vector $x=\left(x_{0}, x_{1}, \ldots, x_{K}\right)$ according to levels. We similarly partition the vectors $b$ and $z$. The matrices $\left\{F_{i}\right\}, i=0,1, \ldots, K$ and $\left\{L_{i}\right\}, i=$ $0,1, \ldots, K$ can now be obtained using the following recur- rence relation:

$$
\begin{aligned}
& F_{0}=A_{0} \\
& y_{0}=b_{0} F_{0}^{-1} \\
& \text { for } i=1 \ldots K \\
& \qquad L_{i-1}=D_{i-1} F_{i-1}^{-1} \\
& \quad F_{i}=A_{i}-L_{i-1} U_{i} \\
& \quad y_{i}=\left(b_{i}-y_{i-1} U_{i}\right) F_{i}^{-1}
\end{aligned}
$$

By backward substitution, one can then find $z_{k}, k=$ $0,1, \ldots, K$ :

$$
\begin{aligned}
& \quad z_{K}=y_{K} \\
& \text { for } i=K-1 \ldots 0 \\
& \quad z_{i}=y_{i}-z_{i+1} L_{i} \\
& \text { end }
\end{aligned}
$$

The block LU factorization algorithm is known to be stable for block tridiagonal matrices that are block diagonally dominant [2]. For the block tridiagonal matrix $P$, the block diagonal dominance condition is (see [2]):

$$
\left\|A_{i}^{-1}\right\|_{1} \geq\left(\left\|U_{i}\right\|_{1}+\left\|D_{i}\right\|_{1}\right), \forall i, 0 \leq i \leq K,
$$

where $U_{0}$ and $D_{K}$ are taken as zero matrices and the 1norm is defined as the maximum row sum. It can then be checked that the matrix $P$ is block diagonal dominant with the two sides of (9) being equal for all $i, 0 \leq i \leq K$. Therefore the algorithm is numerically stable ensuring that $\left\{F_{i}\right\}$ and $\left\{L_{i}\right\}$ will not grow without bounds for large $i$. The algorithm has a computational complexity of $O\left(K W^{3}\right)$ and storage requirement of $O\left(K W^{2}\right)$ which gives rise to significant runtime and storage gains (i.e. gains of order $O\left(K^{2}\right)$ of $O(K)$ respectively) compared to the brute force approach.

We note that a new burst arrival is blocked if one of the following two events happens:

- the Markov chain resides in $(K, j), 0 \leq j \leq W$ (i.e. all wavelength channels are in use)

- the Markov chain resides in state $(i, W), W \leq i<K$ (i.e. all converters are in use) and the incoming wavelength is occupied (this occurs with probability $i / K$ )

The PASTA (Poisson Arrivals See Time Averages) property suggests that the steady-state probabilities at arbitrary times (calculated above) are the same as those of the embedded discrete-time Markov chain at the epochs of arrivals [10]. Therefore, the burst blocking probability $P_{b}$ can mathematically be written as

$$
P_{b}=x_{K} e+\sum_{i=W}^{K-1} x_{i, W} \frac{i}{K}
$$

where $x_{i}=\left(x_{i, 0}, x_{i, 1}, \cdots, x_{i, W}\right)$ for $i \geq W$. 


\section{Phase-type Arrival Process}

It is shown in [5] through simulations that the arrival process characterization has a significant impact on the burst blocking performance. In this section, we generalize our analytical model to cover also the well-known PH-type arrivals which are generalizations of the exponential distribution [12]. It is possible to use PH-type distributions to extend models using exponential distributions to more complex models without losing computational tractability. PHtype models have been extensively used for performance modelling of telecommunication networks [12].

The following analysis is based on [12]. We define a Markov process on the states $\{0,1, \ldots, m\}$ with initial probability vector $(v, \alpha)$ and infinitesimal generator

$$
\left[\begin{array}{ll}
T & t \\
0 & 0
\end{array}\right]
$$

where $v$ is a row vector of size $m$, the subgenerator $T$ is an $m \times m$ matrix, and $t$ is a column vector of size $m$. The distribution of the time till absorption into the absorbing state $m$ is called the $\mathrm{PH}$ distribution with representation $(v, T)$. If the interarrival times are modelled by $\mathrm{PH}$ distributions, then the arrival process is called a PH-type process. In this model successive interarrival times are independent, and therefore it is not possible to model correlated traffic by PH-type arrivals. Models with correlated traffic are generally handled by the (Batch) Markovian Arrival Process ((B)MAP) model and its variants [13],[15]. In this paper we generalize our results to only PH-type arrivals, and the more general correlated arrival case is left for future research.

Let us now study the optical burst switched link with a $\mathrm{PH}$-type arrival process characterized by the pair $(v, T)$. We assume that there is no probability mass for the interarrival times at zero, so we take $\alpha=1-\sum_{i} v_{i}=0$ in this paper. The process $X(t)=\{(i(t), j(t), l(t)): t \geq 0\}$ on the state space $S=\{(i, j, l): 0 \leq i \leq K, 0 \leq j \leq \min (i, W), 0 \leq$ $l \leq m-1\}$, where $i(t)$ and $j(t)$ are defined as before and $l(t)$ is the phase of the burst arrival process at time $t$, is also a CTMC. We enumerate the states as follows:

$$
\begin{aligned}
S= & \{\underbrace{(0,0,0), \cdots,(0,0, m-1)}_{\text {level } 0}, \\
& \underbrace{(1,0,0), \cdots,(1,0, m-1), \cdots,(1,1, m-1)}_{\text {level } 1}, \cdots \\
& \underbrace{(K, 0,0), \cdots,(K, 0, m-1), \cdots,(K, W, m-1)}_{\text {level } K}\}
\end{aligned}
$$

As before, we partition the solution vector $x=$ $\left(x_{0}, x_{1}, \ldots, x_{K}\right)$ according to levels. The resulting process can be shown to have an infinitesimal generator of the same block tridiagonal form (3) where $A_{i}=\left(T-i \mu I_{m}\right) \otimes I_{i+1}$ for $i<W, A_{i}=\left(T-i \mu I_{m}\right) \otimes I_{W+1}+\operatorname{diag}\{-T e\} \otimes \bar{I}_{i}$ for $W \leq i<K$, and $A_{K}=\left(T-T e v-K \mu I_{m}\right) \otimes I_{W+1}$ and $\otimes$ stands for the Kronecker product. Moreover, $D_{i}=$ $\mu I_{m} \otimes \bar{D}_{i}$ and $U_{i}=-T e v \otimes \bar{U}_{i}$. The steady-state probabilities can then be obtained using the block LU factorization algorithm described in the previous section. The blocking probability is then written as

$$
P_{b}=x_{K} e+\sum_{i=W}^{K-1} \sum_{l=0}^{m-1} x_{i, W, l} \frac{i}{K}
$$

where $x_{i}$ is partitioned as $x_{i}=$ $\left(x_{i, 0,0}, x_{i, 0,1}, \cdots, x_{i, 0, m-1}, x_{i, 1,0}, \cdots, x_{i, W, m-1}\right) \quad$ for $i \geq W$.

\section{Numerical Results}

The exact analysis method for calculating blocking probability in OBS nodes introduced in this paper is implemented using Matlab. Without loss of generality, the mean burst time $1 / \mu$ is normalized to unity in all numerical examples presented below.

In the first example, we define the system load $\rho=$ $\lambda / \mu K$ and wavelength conversion ratio $r=W / K$. For three values of $\mathrm{K}(50,100$, and 200), we plot the blocking probability $P_{b}$ as a function of $r$ and $\rho$ in Figure 1 as a 3dimensional mesh. This plot shows that we can obtain loss probabilities for very large systems (e.g., $K=200$ ) and for rare probabilities (e.g.,, $P_{b} \approx 10^{-30}$ ) and for a wide range of system parameters (i.e., $0.4<\rho<1,0<r<1$ ). Moreover, probabilities can be obtained rather rapidly. Plotting one of the three planes on Figure 1 required solution of 1530 problems described in Section 2 and obtaining Figure 1 consisting of three planes took less than an hour on a $2.4 \mathrm{GHz}$ Pentium based PC. We believe that rapid generation of these plots can be very helpful for provisioning purposes.

For the next two figures, we assume Poisson burst arrivals with $\lambda=15$. For a varying number of wavelengths per fiber (i.e., $K$ ), we iteratively find the minimum number of wavelength converters that meets a Grade of Service (GoS) requirement expressed in terms of $P_{b}$. The results are depicted for different values of $P_{b}$ in Figure 2. The region above each curve in Figure 2 represents the region for the set of feasible values $(K, W)$ corresponding to that GoS requirement. For the fixed offered traffic and $P_{b}$, the trade-off between the number of wavelengths and number of converters is illustrated in Figure 2. The lack of converters can be compensated by increasing the number of wavelengths in order to achieve a target GoS.

This trade-off is further investigated in Figure 3 from the OXC cost point of view. Assume a TWC costing $\alpha$ times 


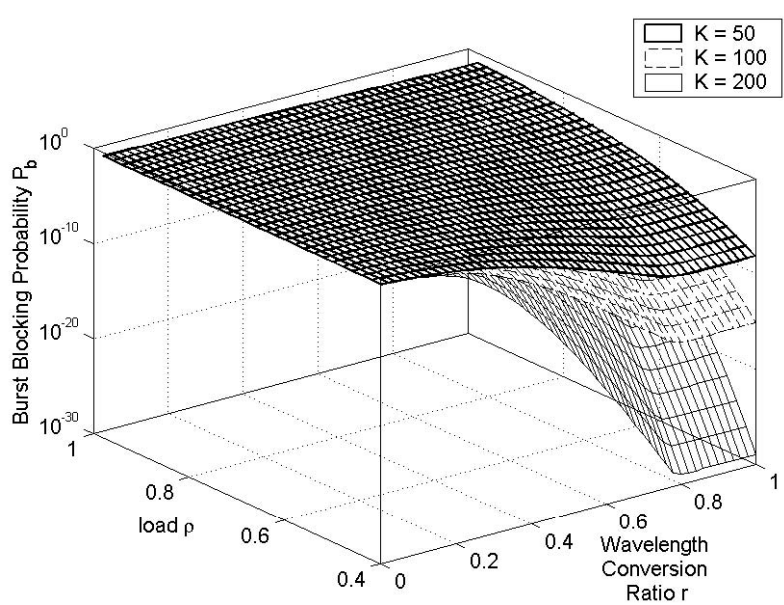

Figure 1. $P_{b}$ as a function of the wavelength conversion ratio $r$ and system load $\rho$ for three different values of $K$

as much as the wavelength channel itself. The points indicated in Figure 3 are the optimal operating points $(K, W)$ for $P_{b}=10^{-4}$ for three different values of $\alpha$. These optimum points correspond to the points where an equi-cost line of slope $-1 / \alpha$ is tangent to the boundary of the feasibility region for $P_{b}=10^{-4}$. As the cost of TWC increases, the optimum operational point moves to a regime where smaller number of converters are used in conjunction with a larger number of wavelengths. The least cost solution has a conversion ratio $r$ of about two thirds when TWCs are relatively inexpensive $(\alpha=2)$. With relatively high cost of conversion $(\alpha=15)$, conversion ratio $r$ reduces to about $10 \%$ in the optimum solution.

The computation times for calculating the blocking probabilities are given in Table 1 for different values of $K$ and $W$. In these computations, we again use Poisson burst arrivals with $\lambda=15$. The results are obtained using Matlab 6.5 on a Pentium- $43 \mathrm{GHz}$ PC. We observe from Table 1 that the blocking probabilities can be calculated within a very short period even for relatively large systems.

Finally, in Figure 4, we present the burst blocking probabilities for $K=100, \mu=1$, and mean interarrival time $=1 / 60$ as a function of the Coefficient of Variation $(\mathrm{CoV})$ $\gamma$ of the interarrival times [8]. The $\mathrm{CoV}$ of a random variable is the standard deviation divided by the mean of that random variable and is indicative of its variability. The case of $\gamma=1$ is for Poisson arrivals, $\gamma<1$ cases are obtained by using an Erlang- $k$ distribution which has $\gamma=1 / k$. The case of $\gamma>1$ is obtained by using a two-phase hyperexponential distribution (i.e. $\mathrm{H}_{2}$ ) with balanced means [8].

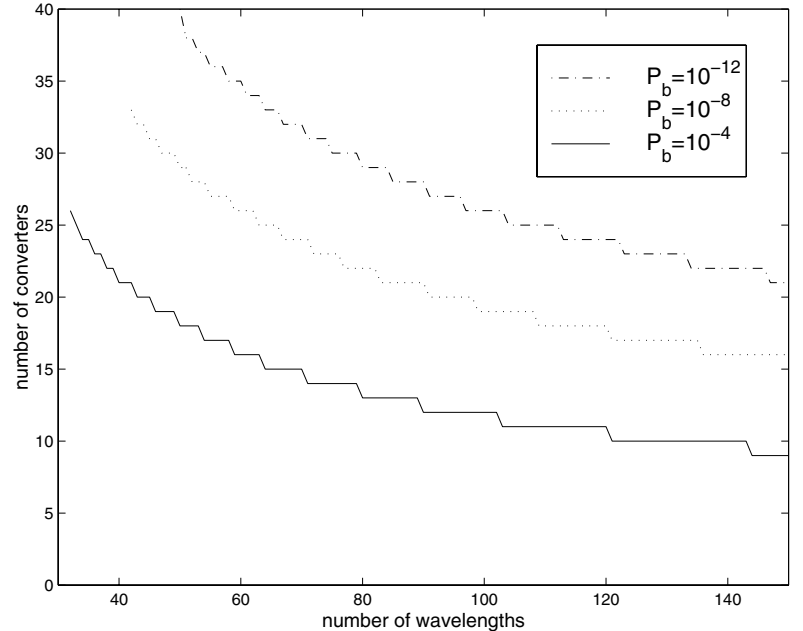

Figure 2. The number of converters required

for a given $P_{b}$ as a function of the number of

wavelength channels

Figure 4 shows that burst blocking probabilities are significantly lower for regularly spaced arrivals (i.e, small coefficient of variation). This shows that the variance (besides the mean) of interarrival times has a substantial impact on burst blocking performance and these second order traffic characteristics also need to be taken into consideration.

\section{Conclusion}

We propose a solution method for exactly calculating blocking probabilities in OBS nodes with partial wavelength conversion. This exact analysis method can be used for efficiently calculating blocking probabilities in large systems, e.g., $K=200$, and rare blocking probabilities, e.g., $P_{b}=10^{-30}$. We show that the method helps in provisioning bufferless OBS links when the cost relationship between a wavelength channel and a converter is available. We also show that the analysis is extendible to phase-type arrivals. Using phase-type arrivals, we find that blocking is reduced when the $\mathrm{CoV}$ of interarrivals is small. We therefore conclude that traffic shaping at the ingress of an OBS network that can reduce the $\mathrm{CoV}$ would also be effective in reducing burst blocking. 


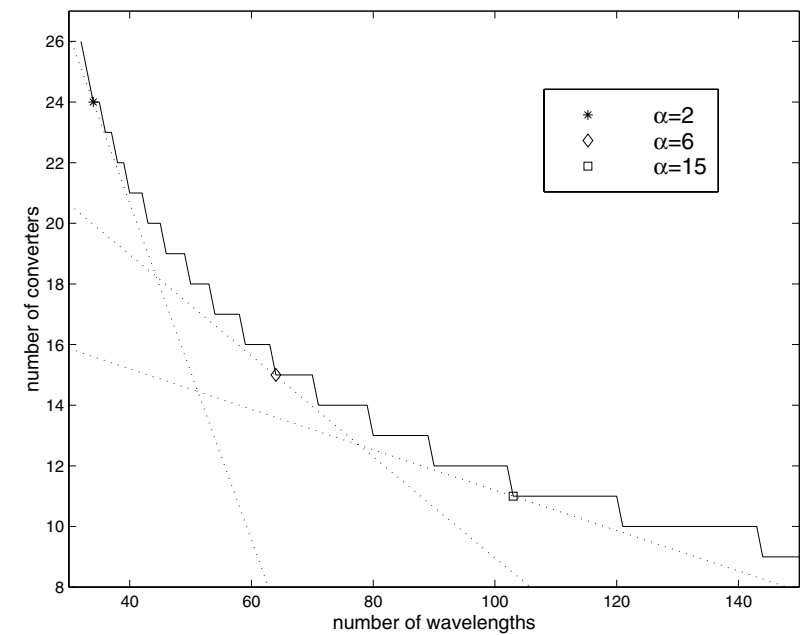

Figure 3. Optimal operating point $(K, W)$ for $P_{b}=10^{-4}$ as a function the cost parameter $\alpha$

\section{References}

[1] R. A. Barry and P. Humblet. Models of blocking probability in all-optical networks with and without wavelenth changers. IEEE J. Sel. Areas Commun., 14:858-867, 1996.

[2] J. Demmel, N. J. Higham, and R. Schreiber. Block LU factorization. Numerical Lin. Alg. and Appl., 2(2):173-190, 1995.

[3] V. Eramo and M. Listanti. Input wavelength conversion in optical packet switches. IEEE Comm. Letters, 7(6):281283, 2003.

[4] V. Eramo, M. Listanti, and P. Pacifici. A comparison study on the wavelength converters number needed in synchronous and asynchronous all-optical switching architectures. IEEE Jour. Lightwave Tech., 21:340-355, 2003.

[5] C. M. Gauger. Performance of converter pools for contention resolution in optical burst switching. In SPIE Optical Networking and Communications Conference (OptiComm 2002), 2002.

[6] G. H. Golub and C. F. van Loan. Matrix Computations. The Johns Hopkins University Press, 3 edition, 1996.

\begin{tabular}{||c||c|c|c|c||}
\hline \hline \multicolumn{1}{||c||}{} & \multicolumn{4}{c||}{$K$} \\
\cline { 2 - 5 }$W$ & 100 & 150 & 200 & 250 \\
\hline \hline 40 & 0.16 & 0.28 & 0.40 & 0.65 \\
\hline 60 & 0.26 & 0.45 & 0.64 & 0.84 \\
\hline 80 & 0.34 & 0.63 & 0.95 & 1.26 \\
\hline 160 & - & - & 2.51 & 3.85 \\
\hline \hline
\end{tabular}

Table 1. Computation times, in seconds, for different values of $K$ and $W$ for $\lambda=15$

[7] D. Gross and C. M. Harris. Fundamentals of Queueing Theory. Wiley, 3rd edition, 1997.

[8] B. R. Haverkort. Performance of Computer and Communication Systems: A Model-based Approach. John Wiley and Sons, 1998.

[9] E. Karasan and E. Ayanoglu. Effects of wavelength routing and selection algorithms on wavelength conversion gain in WDM optical networks. IEEE/ACM Trans. Networking, 6(2):186-196, 1998.

[10] L. Kleinrock. Queuing Systems, Vol. 1, Theory. John Wiley, New York, 1989.

[11] M. Kovacevic and A. Acampora. Benefits of wavelength translation in all-optical clear channel networks. IEEE J. Sel. Areas Commun., 14:868-880, 1996.

[12] G. Latouche and V. Ramaswami. Introduction to matrix analytical methods in stochastic modeling. ASA-SIAM Series on Statistics and Applied Probability, 2002.

[13] D. M. Lucantoni. New results for the single server queue with a batch Markovian arrival process. Stochastic Models, 7:1-46, 1991.

[14] D. Mitra, C. Nuzman, I. Saniee, and P. Whiting. Optical cross-connect with shared wavelength conversion under dynamic load. IEEE/OFC Technical Digest, pages 160-162, 2002. 


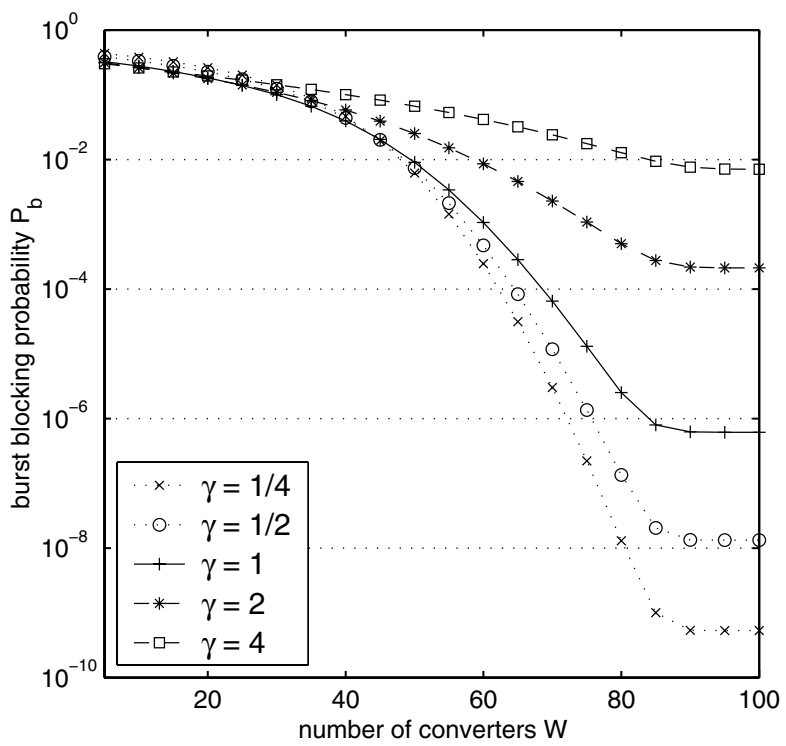

Figure 4. Burst blocking probabilities for $K=$

$100, \mu=1$ and mean interarrival time $=1 / 60$ as a function of $W$ for different values of coefficient of variation $\gamma$

[15] M. F. Neuts. Structured Stochastic Matrices of M/G/l Type and Their Applications. Marcel Dekker, N.Y., 1989.

[16] C. Qiao. Labeled optical burst switching for IP-over-WDM integration. IEEE Communications Magazine, 39(9):104$114,2000$.

[17] C. Qiao and M. Yoo. Optical burst switching (OBS) - a new paradigm for an Optical Internet. Jour. High Speed Networks (JHSN), 8(1):69-84, 1999.

[18] R. Ramaswami and K. N. Sivarajan. Optical Networks: A Practical Perspective. Morgan Kaufmann Publishers, 2 edition, 2002.

[19] J. Spath and S. Bodamer. Routing of dynamic Poisson and non-Poisson traffic in WDM networks with limited wavelength conversion. In 24th European Conference on Optical Communication, 1998.

[20] W. J. Stewart. Introduction to the numerical solution of Markov chains. Princeton University Press, 1994. 